\title{
PHYSICS AND CONTROL: EXPLORING PHYSICAL SYSTEMS BY FEEDBACK
}

\author{
A.L. Fradkov ${ }^{1}$ \\ Institute for Problems of Mechanical Engineering \\ Russian Academy of Sciences \\ 61, Bolshoy, V.O., 199178, St. Petersburg, Russia \\ E-mail: alf@ipme.ru
}

\begin{abstract}
The subject and methodology of an emerging field of cybernetical physics related both to physics and to control are outlined. The aim of a cyberphysical investigation is studying a physical system by means of controlling it. The conservation laws give up their place to the transformation laws. Examples of transformation laws describing the excitability properties of dissipative systems are presented and discussed. Copyright (C) 2001 IFAC
\end{abstract}

Keywords: physics, cybernetics, feedback, resonance, excitability

\section{INTRODUCTION}

Already for more than a decade an enormous growth of interest to the control theory (more broadly - cybernetics) has been observed in the physics community.

For a few previous decades control science and control engineering played only an auxilliary role in physics providing hardware and software design methods for automated experimental equipment (e.g. accelerators) Conventional control goals were formulated in terms of reaching and stabilization of the prespecified experimental regimes.

The breakthrough was initiated by only a few papers. Perhaps, the most influentuable one was that of E. Ott, C. Grebogi and J. Yorke (1990) entitled "Controlling Chaos". The authors manifested that for chaotic systems small feedback changes of a system parameter can result in significant changes

1 The work was supported in part by Russian Foundation of Basic Research (grant RFBR 99-01-00672), Russian Federal Program "Integration"(projects A0151, A0145, 3.1226), Complex Program of the Presidium of RAS "Control of nonlinear mechanical systems under uncertainty and chaos", part 1.4 of the system behavior, e.g. transform chaotic motions into the periodic ones. The idea has become popular in the physics community almost instantaneously. Since 1990 hundreds of papers were published demonstrating ability of small control to change dynamics of the systems significantly, even qualitatively. It is important that the obtained results were interpreted as discovering new properties of the physical systems. By the end of the year 2000 the number of quotations of the paper (Ott et.al., 1990) exceeded 1100 while the total number of the papers related to control of chaos exceeded $2500^{2}$. In a significant number of them some real applications of chaos control in laser and chemical technologies, telecommunications, medicine, etc. are discussed (Arecchi et al., 1998). Similar property of reachability of the control goal by means of arbitrarily small control was observed in a broader class of oscillatory systems (Fradkov and Pogromsky, 1998; Shiriaev and Fradkov, 2001).

\footnotetext{
2 The figures are obtained by search over Science Citation Index (www.isiglobalnet.com), see (Fradkov and Evans, 2001a)
} 
Another example of emerging research field is Control of molecular and quantum systems. Although some theoretical results have been reported previously, see, e.g. (Butkovskii and Samojlenko, 1979, 1984; Huang et. al., 1983; Belavkin, 1983), the real breakthrough was caused by appearance of ultrafast lasers making possible direct control of chemical bonds in the molecules (Zewail, 2000). In some papers real experiments with the Maxwell's Demon (clearly a controller!) have been discussed (Lloyd, 1995). Note that since its creation in 1871, the Maxwell's Demon used to be considered just as a hypothetical being useful for discussing some thought experiments, see (Leff and Rex, 1990). An interest in design of quantum computers has become another powerful stimulus to the development of this field (see DiVincenzo, 1995; Valiev, 2000).

A number of papers published in the field of quantum control was increasing as rapidly as that in the field of control of chaos; some core results were surveyed in (Dahleh et. al., 1997; Rabitz, $2000,2001)$. It is important that the aim of the researches is twofold (Rabitz, 2000):

- to create a particular product that is unattainable by conventional chemical means;

- to achieve a better understanding of atoms and molecules and their interactions.

The third, more narrow stream of researches in the boundary field between physics and control is related to using cybernetical methods for description of dynamics (model building) of physical systems. Such attempts were undertaken both for classical mechanical systems (Bellman and Dreifus, 1964; Fradkov, 1991; Fradkov et. al., 1999) and for quantum-mechanical systems (Rosenbrock, 2000; Grössing, 2000). Cybernetical approach provides new interpretations of the equations of motion, new techniques of their reduction, etc.

The above facts provide evidences for existence of the new emerging field of research related both to physics and to control. It was suggested to call it "Cybernetical Physics" (Fradkov, 1999a; 1999b).

In this paper an attempt to outline some features of this field is made.

\section{CYBERNETICAL PHYSICS: SUBJECT AND METHODOLOGY}

Cybernetical Physics (CP) can be defined as the branch of science aimed at studying physical systems by cybernetical means (by means of control). In order to characterize its subject, i.e. control problems related to $\mathrm{CP}$ one needs to specify classes of controlled plant models, control objec- tives (goals) and admissible control algorithms. Besides, one needs to describe the methodology of CP, i.e. classify main methods uses for solving the problems and characterize typical results in the field. A brief description of the subject and methodology of CP will be done below.

\subsection{Models of controlled systems.}

A formal statement of any control problem begins with a model of the system to be controlled (plant) and a model of the control objective (goal). Even if the plant model is not given (like in many real world applications) it should be determined in some way. Several classes of models are considered in the literature related to control of physical systems. The most common class consists of continuous systems with lumped parameters described in state space by differential equations

$$
\dot{x}=F(x, u),
$$

where $x$ is $n$-dimensional vector of the state variables; $\dot{x}=d / d t$ stands for the time derivative of $x ; u$ is $m$-dimensional vector of inputs (control variables). Vector-function $F(x, u)$ is usually assumed continuously differentiable. It is important to note that model (1) encompasses two physically different cases:

A. The input variables represent some physical variables (forces, torques, intensity of electrical or magnetic fields, etc.) For example a model of a controlled oscillator (pendulum) can be put into the form

$$
J \ddot{\varphi}+r \dot{\varphi}+m l \sin \varphi=u(t)
$$

where $\varphi$ is the angle of deflection from vertical; $J, m, l$ are physical parameters of the pendulum (inertia, mass, length); $\mathrm{u}(\mathrm{t})$ is a controlling torque. The description (2) is transformable into the form (1) with the state vector $x=(\varphi, \dot{\varphi})^{T}$.

B. The input variables represent change of physical parameters of the system, i.e. $u(t)=p-p_{0}$, where $p_{0}$ is the nominal value of the physical parameter $p$. For example, let the pendulum be controlled by changing its length. Then the model, instead of (2) becomes

$$
J \ddot{\varphi}+r \dot{\varphi}+m\left(l_{0}+u(t)\right) \sin \varphi=0,
$$

where $l_{0}$ is initial length of the pendulum.

Although in some papers the difference between the cases A and B is emphasized, for the purpose of studying the nonlinear system (1) the difference is not important. 3

\footnotetext{
3 It makes sense to treat differently the case of control variables and the case of control parameters for linear
} 
If external disturbances are present, we need to consider more general time-varying models

$$
\dot{x}=F(x, u, t) .
$$

On the other hand, many nonlinear control problems can be described using more simple affine in control models

$$
\dot{x}=f(x)+g(x) u .
$$

The model should also include the description of measurements, i.e. the $l$-dimensional vector of output variables $y$ should be defined, for example

$$
y=h(x) .
$$

If the outputs are not defined explicitly, it will be assumed that all the state variables are available for measurement, i.e. $y=x$.

Many authors deal with discrete-time state-space models

$$
x_{k+1}=F_{d}\left(x_{k}, u_{k}\right) \text {. }
$$

where $x_{k} \in \mathbb{R}^{n}, u_{k} \in \mathbb{R}^{m}, y_{k} \in \mathbb{R}^{l}$, are state, input and output vectors at $k$ th stage of the process. Then the model will be defined by the mapping $F_{d}$. A lot of publications are devoted to control of distributed systems: delay-differential and delay-difference models, spatio-temporal systems described by partial differential equations or their discrete analogues, etc.

\subsection{Control goals}

The typical goal for control of chaotic systems is stabilizing of an unstable periodic solution (orbit). Let $x_{*}(t)$ be the $T$-periodic solution of the free (uncontrolled, i.e. $u(t)=0$ ) system (1) with initial condition $x_{*}(0)=x_{* 0}$, i.e. $x_{*}(t+T)=x_{*}(t)$ for all $t \geq 0$. If the solution $x_{*}(t)$ is unstable it is reasonable to pose the goal as stabilization in some sense, e.g. driving solutions $x(t)$ of (1) to $x_{*}(t)$

$$
\lim _{t \rightarrow \infty}\left[x(t)-x_{*}(t)\right]=0
$$

or driving the output $y(t)$ to the desired output function $y_{*}(t)$, i.e.

$$
\lim _{t \rightarrow \infty}\left[y(t)-y_{*}(t)\right]=0
$$

for any solution $x(t)$ of (1) with initial conditions $x(0)=x_{0} \in \Omega$, where $\Omega$ is given set of initial conditions.

systems because the linear system with linear parametric feedback control leaves the class of linear systems (becomes binlinear). However the class of nonlinear systems (1) is closed with respect to all nonlinear feedbacks.
The problem is to find a control function in the form of open loop (feedforward) control

$$
u(t)=U\left(t, x_{0}\right)
$$

in the form ofstate feedback

$$
u(t)=U(x(t))
$$

or in the form ofoutput feedback

$$
u(t)=U(y(t))
$$

to ensure the goal (8) or (9).

Such a problem is nothing but a tracking problem, very familiar to control theorists. However the key feature of the control of chaotic systems as claimed by Ott et.al., (1990) is to achieve the goal by means of sufficiently small (ideally, arbitrarily small) control. Solvability of this task is not obvious since the trajectory $x_{*}(t)$ is unstable.

A special case of the above problem is stabilization of the unstable equilibrium $x_{* 0}$ of system (1) with $u=0$, i.e., stabilization of $x_{* 0}$, satisfying $F\left(x_{* 0}, 0\right)=0$. Again, it looks like a standard regulation problem with an additional restriction that we seek for "small control" solutions. However, such a restriction makes the problem far from standard: even for a simple pendulum, nonlocal solutions of the stabilization problem with small control were obtained only recently, see (Shiriaev and Fradkov, 2001). The class of admissible control laws can be extended by introducing dynamic feedback described by differential or time-delayed models. Similar formulations hold for discrete and time-delayed systems.

Second class of control goals corresponds to the problems of excitation or generation of chaotic oscillations (also called chaotization or anticontrol). Formally these problems can be reduced to the form (9), but the goal trajectory $x_{*}(t)$ is no longer periodic, while the initial state is equilibrium. The goal trajectory may be specified only partially. Instead, a scalar goal function $G(x)$ is given and the goal is to achieve the limit equality

$$
\lim _{t \rightarrow \infty} G(x(t))=G_{*}
$$

or inequality

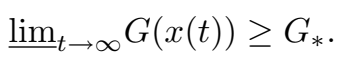

In many cases the total energy of mechanical or electrical oscillations can serve as $G(x)$.

Third important class of control goals corresponds to synchronization (more accurately, controlled synchronization in opposition to autosynchronization or self-synchronization. Generally speaking, synchronization is understood as concordance or 
concurrent change of the states of two or more systems or, perhaps, concurrent change of some quantities related to the systems, e.g. equalizing of oscillation frequencies. If the required relation is established only asymptotically, one speaks about asymptotic synchronization. If synchronization does not exist in the system without control ( for $u=0$ ) we may pose the problem as finding the control function which ensures synchronization in the closed-loop system, i.e. synchronization may be a control goal. For example the goal corresponding to asymptotic synchronization of the two system states $x_{1}$ and $x_{2}$ can be expressed as follows:

$$
\lim _{t \rightarrow \infty}\left[x_{1}(t)-x_{2}(t)\right]=0
$$

In the extended state space $x=\left\{x_{1}, x_{2}\right\}$ of the overall system, relation (15) implies convergence of the solution $x(t)$ to the diagonal set $\left\{x: x_{1}=\right.$ $\left.x_{2}\right\}$. Asymptotic identity of the values of some quantity $G(x)$ for two systems can be formulated as

$$
\lim _{t \rightarrow \infty}\left[G\left(x_{1}(t)\right)-G\left(x_{2}(t)\right)\right]=0
$$

Often it is convenient to rewrite the goals (8), $(9),(13),(15)$ or (16) in terms of appropriate goal function $Q(x, t)$ as follows:

$$
\lim _{t \rightarrow \infty} Q(x(t), t)=0
$$

For example to reduce goal (15) to the form (17) one may choose $Q(x)=\left|x_{1}-x_{2}\right|^{2}$. Instead of Euclidean norm other quadratic functions can also be used. E.g. for the case of the goal $(8)$ the goal function $Q(x, t)=\left[x-x_{*}(t)\right]^{T} \Gamma\left[x-x_{*}(t)\right]$, where $\Gamma$ is positive definite symmetric matrix can be used. The freedom of choice of the goal function can be utilized for design purposes.

Finally, the goals may be specified as modification of some quantitative requirements to the limit behavior of the system, i.e. changing fractal dimension of its attractor.

Some of the above mentioned goals are not typical for conventional control theory because they do not specify the desired behavior of the system completely. These classes of control problems belong to the area of the so called partial control which development has been activized recently (Vorotnikov, 1998; Fradkov et.al., 1999). It is important, that the above goals should be achieved without significant intervening the system dynamics, i.e. the control algorithms to be designed should meet the small control or weak control requirement. Therefore typical formulations of the control problems look as follows:
- find all the behaviors that can be attained by the control functions of the given (sufficiently small) norm;

- find on control function (feedback operator) of minimum norm ensuring the given control goal.

Of course, traditional formulations are not ruled out and may appear in some physical problems.

\subsection{Methodology}

The methodology of cybernetical physics is based on the control theory. Typically, some parameters of physical systems are unknown and some variables are not available for measurement. From the control viewpoint it means that control design should be performed under significant uncertainty, i.e. methods of robust or adaptive control should be used. A variety of design methods have been developed both for linear and for nonlinear systems (Zhou et al, 1996; Krstić et al, 1995; Fradkov et al, 1999, etc.). Methods of partial control and weak control are also available (Vorotnikov, 1998; Fradkov and Pogromsky, 1998).

\subsection{Results}

A great deal of results in physics are presented in form of conservation laws, stating that some quantities do not change during evolution of the system. However, the formulations in $\mathrm{CP}$ are different. The results in CP establish how the evolution of the system can be changed by control. Therefore the results in CP should be formulated as transformation laws, specifying the classes of changes in the evolution of the system attainable by the given class of controls, i.e. specifying the limits of control. Typical example of transformation law: "Any chaotic trajectory can be transformed into a periodic one by means of control" (Ott et al. 1990). Some other examples related to excitation of a system with small control are presented below.

\section{FEEDBACK RESONANCE AND EXCITABILITY OF SYSTEMS}

\subsection{Feedback resonance}

The concept of resonance has numerous applications in physics and mechanics. Its essence is that small resonant force applied to a system leads to significant changes in system behavior. First clear description of resonance phenomenon was given by Galileo Galilei in "Discorsi a Dimostrazioni Matemaci" (1638): “... Pendulum at rest although very heavy, can be put into motion, 
and very significant if we stop our breath when it is coming back and blow again at the instance, corresponding to its swing".

The resonance phenomenon is well understood and perfectly studied for linear systems. If, however, the dynamics of the system is nonlinear, the resonance is much more complicated because interaction of different harmonic signals in nonlinear system may create complex and even chaotic behavior (Thompson and Stewart, 1986). The reason is, roughly speaking, in that the natural frequency of a nonlinear system depends on the amplitude of oscillations.

In (Fradkov,1999a, 1999b) the idea was pursued to create resonance in a nonlinear oscillator by changing the frequency of external action as a function of oscillation amplitude. Consider a systems described by state-space equations

$$
\dot{x}=F(x, u), y=h(x)
$$

where $x \in R^{n}$ is state vector, $u, y$ are scalar input and output, respectively.

To make this idea realizable, $u(t)$ should depend on the state of the system $x(t)$ or on the current measurements $y(t)$, which exactly means introducing a state feedback

$$
u(t)=U(x(t))
$$

or output feedback

$$
u(t)=U(y(t)) .
$$

Now the problem is: how to find the feedback law (19) or (20) in order to achieve the maximum limit amplitude of output?

In (Fradkov, 1999b) this problem was formulated as that of optimal control. We may pose it as finding

$$
\begin{gathered}
Q(\gamma)=\limsup |y(t)|^{2} \\
|u(s)| \leq \gamma, \\
0 \leq s \leq t \\
x(0)=0 \\
t \geq 0
\end{gathered}
$$

We assume that the system (18) is BIBO stable and $x=0$ is equilibrium of the unforced system ( $F(0,0)=0, h(0)=0)$ in order to ensure $Q(\gamma)$ to be well defined. Apparently, the signal providing maximum excitation should depend not only on time but also on system state, i.e. input signal should have a feedback form. Note that for linear systems the value of the problem (21) depends quadratically on $\gamma$. Therefore it is naturally to introduce the excitability index (EI) for the system (18) as follows:

$$
E(\gamma)=\frac{1}{\gamma} \sqrt{Q(\gamma)}
$$

where $Q(\gamma)$ is the optimum value of the problem (21). It is clear that for linear asymptotically stable systems $E(\gamma)=$ const. For nonlinear systems $E(\gamma)$ is a function of $\gamma$ that characterizes excitability properties of the nonlinear system. It was introduced in (Fradkov, 1999b) with respect to the energy-like output. For MIMO systems excitability indices $E_{i j}$ can be introduced in a similar way for every pair of input $u_{i}$ and output $y_{j}$. The concept of EI is related to the concept of input-output (I-O) gain If I-O gain exists, it provides an upper bound for EI. Conversely, if EI is finite, it estimates the minimal value of I-O gain.

The solution to the problem (21) is quite complicated in most cases. However we can use approximate locally optimal or speed-gradient solution

$$
u(x)=\gamma \operatorname{sign}\left(g(x)^{T} \nabla h(x) h(x)\right),
$$

where $g(x)=\left.\frac{\partial F(x, u)}{\partial u}\right|_{u=0}$, obtained by maximizing the principal part of instant growth rate of $|y(t)|^{2}$. It follows from the results of Chernousko (1968) that for small $\gamma$ the value of $|y(t)|$ achievable with input (23) for sufficiently large $t \geq 0$ differs from the optimal value $Q(\gamma)$ by the amount of order $\gamma^{2}$. An important consequence is that excitability index can be estimated directly by applying input (23) to the system. For real world systems it can be done experimentally. Otherwise, if a system model is available, computer simulations can be used.

\subsection{Properties of excitability index}

Since excitability index of a system characterizes its sensitivity to a feedback excitation, it is important to relate excitability to inherent dymanical properties of a system. Such kind of bounds for a class of strictly passive systems (systems with full dissipation) are established below. To simplify formulations we will study the averaged excitability index defined as $\bar{E}(\gamma)=\frac{1}{\gamma} \sqrt{\bar{Q}(\gamma)}$, where

$$
\bar{Q}(\gamma)=\sup _{\substack{|u(s)| \leq \gamma \\ 0 \leq s \leq t, t \geq 0 \\ x(0)=0}} \frac{1}{t} \int_{0}^{t} y^{2}(s) d s .
$$

Proposition 1. Let the controlled system (24) be output strictly passive, i.e. there exist functions $V(x) \geq 0, \varrho(y) \geq 0$ and positive numbers $\alpha, \varrho_{0}, \varrho_{1}$ such that $\varrho_{0}|y|^{2} \leq \varrho(y) \leq \varrho_{1}|y|^{2}, V(0)=0$ and 


$$
\begin{aligned}
& V(x(t))-V(x(0)) \\
& =\int_{0}^{t}[u(s) y(s)-\varrho(y(s))] d s .
\end{aligned}
$$

Then the following inequality holds for $x(0)=0$ and all $t \geq 0$ :

$$
\int_{0}^{t}\left[y(s)^{2}\right] d s \leq \varrho_{0}^{-2} \int_{0}^{t}\left[u(s)^{2}\right] d s .
$$

If, additionally, $|u(t)| \leq \gamma$, then

$$
\frac{1}{t} \int_{0}^{t}\left[y(s)^{2}\right] d s \leq\left(\frac{\gamma}{\varrho_{0}}\right)^{2}
$$

and, therefore, $\bar{E}(\gamma) \leq \varrho_{0}^{-1}$. Besides, if $V(x) \geq$ $\alpha|y|^{2}$, then

$$
\lim _{t \rightarrow \infty} \sup _{\substack{|u(s)| \leq \gamma \\ 0 \leq s \leq t, x(0)=0}} V(x(t)) \geq \alpha\left(\frac{\gamma}{\varrho_{1}}\right)^{2} .
$$

We see that the action (23) creates a sort of resonance mode in a nonlinear system: for weakly damped systems even a small action having form (23) leads to large oscillations of the output and can insert a substantial amount of energy into the system.

\subsection{Case of Euler-Lagrange systems}

The above bounds are expressed in terms of the ratio "(excitation amplitude)/(dissipation)." Simple examples show that it is not possible to obtain a lower bound similar to (28) for arbitrary output of the system. However it is possible for a class of physical systems described by Euler-Lagrange equations with dissipative forces, if the output is specified as the energy of the system.

Consider an Euler-Lagrange system

$$
A(q) \ddot{q}+R(\dot{q})+\nabla \Pi(q)=u,
$$

where $q \in \mathbb{R}^{m}$ is vector of generalized coordinates, $u=u(t) \in \mathbb{R}^{m}$ is vector of controlling forces (torques), $A(q)$ is matrix of kinetic energy, $\Pi(q)$ is potential energy, $R(\dot{q})$ is vector of dissipative forces. Let $\mathcal{H}(q, \dot{q})$ be total energy of the system:

$$
\mathcal{H}(q, \dot{q})=\frac{1}{2} \dot{q}^{T} A(q) \dot{q}+\Pi(q) .
$$

Introduce upper and lower excitability indices $E^{+}(\gamma), E^{-}(\gamma)$ as follows

$$
E^{ \pm}(\gamma)=\frac{1}{\gamma} \sqrt{\chi^{ \pm}(\gamma)}
$$

where

$$
\begin{aligned}
& \chi^{+}(\gamma)=\varlimsup_{t \rightarrow \infty} \sup _{\substack{|u(\cdot)| \leq \gamma \\
x(0)=0}} \mathcal{H}(q(t), \dot{q}(t)), \\
& \chi^{-}(\gamma)=\lim _{t \rightarrow \infty} \sup _{\substack{u(\cdot) \mid \leq \gamma \\
x(0)=0}} \mathcal{H}(q(t), \dot{q}(t)),
\end{aligned}
$$

Proposition 2. Let

$$
\begin{gathered}
0<\alpha^{-} \leq \lambda_{i}(A(q)) \leq \alpha^{+}, \\
\varrho^{-}|\dot{q}|^{2} \leq R(\dot{q})^{T} q \leq \varrho^{+}|\dot{q}|^{2}, \\
0 \leq \Pi(q) \leq d .
\end{gathered}
$$

Then

$$
\begin{aligned}
& \frac{\alpha^{-}}{2}\left(\frac{\gamma}{\varrho^{+}}\right)^{2} \leq \chi^{-}(\gamma) \\
& \quad \leq \chi^{+}(\gamma) \leq m \alpha^{+}\left(\frac{\gamma}{\varrho^{-}}\right)^{2}+d
\end{aligned}
$$

Corollary. If $R(\dot{q})=\varrho \dot{q}$ and $\varrho \rightarrow 0$, then

$$
E^{ \pm}(\gamma) \sim \frac{C_{ \pm}}{\varrho} .
$$

Remark. Locally optimal control is

$$
u=\gamma \operatorname{sign}(\dot{q}) \text {. }
$$

It will be suboptimal for small $\gamma>0$. Action (36) creates a resonance regime of the system (29) because the order of the estimate (35) coincides with the order of magnitude frequency response for linear systems. Relations (34) or (35) can be considered as transformation of energy laws for dissipative Euler-Lagrange systems.

\section{CONCLUSIONS}

The growth of interaction between physics and control is imperative of time. Physics is actively seeking for new tools and frameworks adequate to new classes of problems. In the second half of XXth century the apparatus of dynamical systems theory and information technologies were incorporated. It seems it is the turn of the control theory.

Finding mutual understanding between two sciences faces the inherent difference of their structures. Indeed, physics (particularly, mechanics) is a classical descriptive science and its aim is to describe the natural behavior of the system. The control science, in the contrary, is a paradigme of perscriptive science with the main aim to perscribe the desired behavior to the system (Brockett, 1977). To achieve cooperation, both sides are making some steps: physics admits intervening the natural system by a small dose of control, while control theory starts studying problems with incompletely specified goal behavior (partial control problems). 
In the nearest future one may expect appearance of new fruits of this compromise.

\section{REFERENCES}

Arecchi, F.T., S. Boccaletti, M. Ciofini, R. Meucci and C. Grebogi (1998). The control of chaos: Theoretical schemes and experimental realizations. Intern. J. Bifurcations and Chaos, 8, 1643-1655.

Belavkin, V.P. (1983) Autom. Remote Control, 44, 178-188.

Bellman, R. and S.E. Dreyfus (1964). Applied Dynamic Programming. Princeton Univ. Press.

Blekhman, I.I. (2000). Vibrational Mechanics. World Scientific, Singapore.

Brockett, R.W. (1977). Control theory and analytical mechanics. In: Geometric Control Theory, Lie Groups, v. VII. / Eds. C. Martin, R. Hermann. Mat. Sci. Press, Brookine, MA, pp. 1-48.

Butkovskii, A.G. and Yu.I. Samoilenko (1979). Control of quantum-mechanical systems, I, II. Autom. Remote Control, 40 (4,5).

Butkovskii, A.G. and Yu.I. Samoilenko (1984). Control of Quantum-mechanical Processes. Nauka, Moscow. (English translation: Kluwer Academic Publishers, Dordrecht, 1990).

Chen, Yu, P. Gross, V. Ramakrishna, H. Rabitz, K. Mease and H. Singh (1997) Control of classical regime molecular objectives - applications of tracking and variations of the theme. Automatica, (9), 1617-1633.

Chernousko F.L. (1968). Some problems of optimal control with a small parameter. J. Appl. Math. Mech., (3), 12-22.

Dahleh, M., A. Pierce, H. Rabitz and V. Ramakrishna (1996). Control of molecular motion. Proc. IEEE, 84(1), 7-15.

DiVincenzo, D.P. (1995). Quantum computation. Science, 270, 255-261.

Fradkov, A.L. (1991). Speed-gradient laws of control and evolution, Prepr. 1st European Control Conf., Grenoble, pp.1865-1870.

Fradkov, A.L. (1999). Exploring nonlinearity by feedback. Physica D., 128(2-4), 159-168.

Fradkov, A.L.(1999) Investigation of physical systems by means of feedback. Autom. Remote Control, 60(3), 3-22.

Fradkov, A.L.(1999). Feedback resonance in nonlinear oscillators. Proc. 5th Europ. Contr. Conf., Karlsruhe.

Fradkov, A.L. (2000). A nonlinear philosophy for nonlinear systems. Proc. 39th IEEE Conf. Decisions and Control, Sydney, 12-15 Dec. 2000, pp. 4397-4402.

Fradkov, A.L. and R. Evans (2001). Control of chaos: some open problems (submitted).

Fradkov, A.L., I.V. Miroshnik and V.O. Nikiforov (1999). Nonlinear and Adaptive Control of
Complex Systems. Kluwer Academic Publishers, Dordrecht.

Fradkov, A.L. and A.Yu. Pogromsky (1998). Introduction to Control of Oscillations and Chaos. World Scientific, Singapore.

Grössing, G. (2000). Quantum Cybernetics. Springer, New York.

Huang, G.M., T.J. Tarn and J.W. Clark (1983). J. Math. Phys., 24, 2608.

Krstić, M., I. Kanellakopoulos and P.V. Kokotović (1995). Nonlinear and Adaptive Control Design, Wiley, New York.

Leff, H.S. and A.F. Rex (Eds) (1990). Maxwell's Demon - Entropy, Information, Computing. Random Hause, New York.

Lloyd, S. (1997). Quantum-mechanical Maxwell's Demon. Phys. Rev., A56(5), 3374-3382.

Ott, T., C. Grebogi and G. Yorke (1990). Controlling chaos. Phys. Rev. Lett., 64(11), 11961199.

Rabitz, H. (2000). Algorithms for closed loop control of quantum dynamics. Proc. 39th IEEE Conf. Decisions and Control, Sydney, 12-15 Dec. 2000, pp.937-942.

Rabitz, H. (2001). Controlling Quantum Phenomena: The Dream is Becoming a Reality Preprints of the 5th IFAC Symp. Nonlin. Contr. Systems (NOLCOS'01). St.Petersburg, 4-6 July, 2001.

Rosenbrock, H.H. (2000). Doing quantum mechanics with control theory. IEEE Trans. Autom. Contr., AC-45(1), 73-77.

Shiriaev, A.S. and A.L. Fradkov (2001). Stabilization of invariant sets for nonlinear systems with application to control of oscillations. Intern. J. of Robust and Nonlinear Control, 11, $215-240$.

Thompson, J.M.T. and H.B. Stewart (1986). Nonlinear Dynamics and Chaos. Wiley, New York.

Valiev, K.A. and A.A. Kokin (2000). Quantum computer: a dream or reality? Regular and Chaotic Dynamics, Izhevsk (in Russian).

Vorotnikov, V.I. (1998). Partial Stability and Control. Birkhäuser, Boston.

Zewail, A. (2000). Femtochemistry: Atomic-Scale dynamics of the chemical bond. (Adapted from the Nobel Lecture). J. Phys. Chemistry, A 2000, 104, 5660-5694.

Zhou, K., J.C. Doyle and K. Glover (1996). Robust and Optimal Control. Prentice Hall. 